\title{
Coupling Between Component Sizing and Regulation Capability in Microgrids
}

\author{
Tulga Ersal, Changsun Ahn, Diane L. Peters, John W. Whitefoot, Abigail R. Mechtenberg, Ian A. Hiskens, \\ Huei Peng, Anna G. Stefanopoulou, Panos Y. Papalambros, and Jeffrey L. Stein
}

\begin{abstract}
Increasing energy security and reliability concerns are intensifying the interest in microgrids. In this setting, design optimization is vital to achieve a reliable infrastructure without overbuilding. This paper considers the impact of frequency and voltage regulation on the optimal design of a conceptual, autonomous military microgrid. This microgrid comprises a solar panel and vehicles as power sources, with each vehicle incorporating a battery and generator. The power output and terminal voltage of these inverter-based sources must be regulated. The paper investigates the effects of battery DC voltage variations on a decentralized regulation scheme, and the resulting influence on optimal component sizing. To this end, controllers are first designed based on the typical assumption that the voltage on the DC side of each inverter is constant. The battery internal resistance is then considered and its impact on regulation performance is investigated. The results show that the battery internal resistance can affect the performance of both frequency and voltage regulation, and consequently must be taken into account in component sizing decisions. Thus, the paper identifies an important coupling between regulation and component sizing problems through battery characteristics, and highlights the need for a combined sizing and regulation framework for microgrid design.
\end{abstract}

Index Terms-Batteries, frequency control, inverters, microgrids, voltage control.

\section{INTRODUCTION}

$\mathbf{M}$ ICROGRIDS are collections of electrical loads and micro-sources functioning as a single system that can operate either in connection with a larger power grid

Manuscript received August 22, 2012; revised February 08, 2013; accepted April 15,2013. Date of publication July 15, 2013; date of current version August 21, 2013. This work was supported by the Automotive Research Center (ARC), a U.S. Army Center of Excellence in Modeling and Simulation of Ground Vehicles. Paper no. TSG-00520-2012.

T. Ersal (corresponding author), H. Peng, A. G. Stefanopoulou, P. Y. Papalambros, and J. L. Stein are with the Department of Mechanical Engineering, University of Michigan, Ann Arbor, MI 48109 USA (e-mail: tersal@umich. edu; hpeng@umich.edu; annastef@umich.edu; pyp@umich.edu; stein@umich. edu).

C. Ahn was with the University of Michigan, Ann Arbor, MI 48109 USA. He is now with the Department of Magnetic Levitation and Linear Drive, Korea Institute of Machinery and Materials, Daejeon, Korea (e-mail: sunahn@kimm.re. $\mathrm{kr})$.

I. A. Hiskens is with the Department of Electrical Engineering and Computer Science, University of Michigan, Ann Arbor, MI 48109 USA (e-mail: hiskens@umich.edu).

D. L. Peters was with the University of Michigan, Ann Arbor, MI 48109 USA. She is now with LMS, A Siemens Business, Troy, MI 48098 USA (e-mail: diane.peters@1msintl.com).

J. Whitefoot was with the University of Michigan, Ann Arbor, MI 48109 USA. He is now with the National Highway Traffic Safety Administration, Washington, DC 20590 USA (e-mail: whitefootj@gmail.com).

A. Mechtenberg was with the University of Michigan, Ann Arbor, MI 48109 USA. She is now with Franklin W. Olin College of Engineering, Energy Systems, Needham, MA 02492 USA (e-mail: abigail.mechtenberg@olin.edu).

Please address all correspondence to T. Ersal, corresponding author (e-mail: tersal@umich.edu).

Color versions of one or more of the figures in this paper are available online at http://ieeexplore.ieee.org.

Digital Object Identifier 10.1109/TSG.2013.2260363 or completely autonomously [1]. They have been attracting much research interest due to their potential to increase energy security and reliability, as well as foster the penetration of distributed renewable resources (e.g., wind, solar) and distributed storage (e.g., plug-in vehicles, community energy storage) [2]-[11]. Because of this potential, the U.S. Department of Defense (DoD) is interested in microgrids as indicated by the SPIDERS (Smart Power Infrastructure Demonstration for Energy Reliability and Security) project, which aims to demonstrate the first complete DoD installation with a secure microgrid capable of islanding.

Whereas the SPIDERS project presents an example microgrid that can operate in both grid-connected and islanded mode, forward operating bases (FOBs) - military bases temporarily established to support tactical operations-exemplify the need for maximizing operational autonomy. Traditionally, these microgrids have relied entirely on diesel generators, whose transportation and fuel re-supply increase the vulnerability of the FOB and the supply lines themselves. Indeed, electric power generation can account for over $70 \%$ of fuel consumption at or near the tactical edge [12], and the U.S. Army indicates that $50 \%$ of the casualties during resupply missions in Iraq and Afghanistan are due to fuel delivery [13].

To make FOBs more autonomous, the microgrid can leverage local renewable resources and military vehicles with on-board electrical generation and energy storage capability. Such a concept FOB considered in this paper is shown in Fig. 1. Instead of relying entirely on stationary generators, it integrates into the microgrid a solar panel and a fleet of vehicles, each with a battery and on-board generator. Military vehicles in a FOB require significant on-board electric power generation and storage to supply electrical equipment such as radars, radios, and computers. For strategic reasons, it is necessary to supply this power with either minimum idling of the main propulsion engines or in silent watch.

With this military application in mind, this paper is concerned with optimal design of microgrids considering two different aspects: component sizing and regulation. One important aspect of microgrid design is the sizing of its components. While component sizing is often performed based on the expected peak load of the microgrid, it has also been recognized that this approach may be highly conservative and sub-optimal, as many components will be larger than necessary. Power dispatch control strategies can be used to reduce this conservatism, but at the cost of coupling the optimal sizing and dispatch problems [14]-[23]. On the other hand, the regulation problem focuses on controlling the voltage and frequency in the microgrid.

Two types of sources in the example microgrid, the solar panel and the batteries in the plug-in vehicle fleet, are DC and need an inverter interface to connect to an AC microgrid (e.g., 


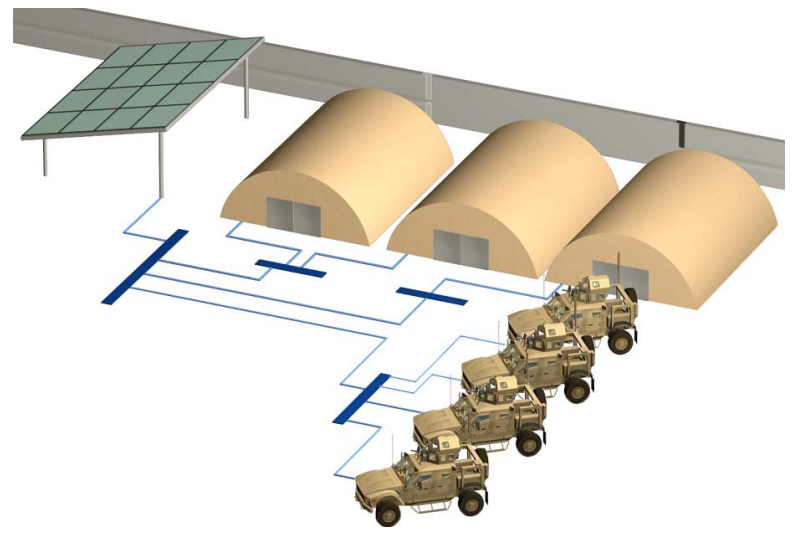

Fig. 1. Example conceptual military microgrid considered in this study for a forward operating base (FOB). The power sources consist of a solar panel array and a fleet of electrified vehicles.

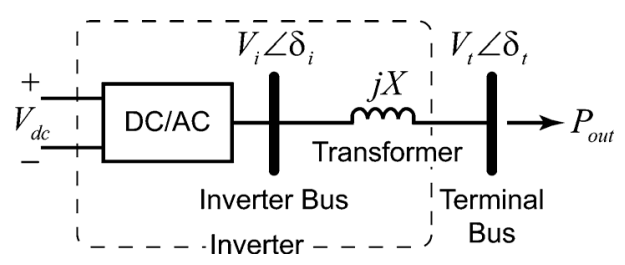

Fig. 2. Inverter-grid interface model.

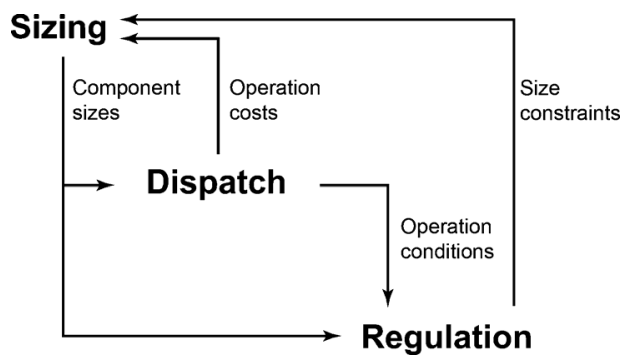

Fig. 3. The integrated optimal microgrid design framework employed in this study.

see Fig. 2). Controlling the inverter is critical to regulating the $\mathrm{AC}$ voltage and power, as well as to establishing the microgrid frequency during autonomous operation [24], [25]. Many techniques have been proposed and used to achieve this goal [6], [10], [11], [24]-[27]. However, coupling between the optimal sizing and regulation problems has not yet been considered.

The goal of this paper is to explore the nature of such couplings. This is achieved by creating an integrated framework in which the sizing, dispatch, and regulation problems are optimally solved in a nested manner (Fig. 3). The component sizing is done first with no regard to regulation, but by considering the optimal dispatch. This initial solution to the component sizing and dispatch problem is then used to define the regulation problem. The outcome of the regulation problem is a new set of design constraints that were originally not included in the optimal component sizing problem. The component sizing problem is then solved again, and the process is iterated. This framework is applied to the military microgrid example in Fig. 1 as a case study to show that the coupling between the component sizing and regulation problems can indeed be significant.

The paper is organized as follows. The results of the initial optimal sizing and dispatch study for the example microgrid are first summarized. This lays out the scenario for the subsequent regulation study. The paper then focuses on this regulation problem. A model for the example microgrid is developed and a fixed-structure controller is proposed to manage the power setpoints of the battery and generator. The initial control design assumes that the battery is a constant voltage source. Then, the battery is modeled as a voltage source in series with a resistor to approximate the battery internal resistance and its power limitation. With this model, the effect of the battery resistance, or power availability, on frequency and voltage regulation is investigated. Moreover, the internal resistance of a battery of a particular chemistry is considered to further illustrate the extent to which regulation considerations can affect the component sizing problem. Hence, a coupling is shown between the fast-time-scale regulation and slow-time-scale sizing problems, which traditionally have been studied independently due to the disparity of their time scales.

\section{Microgrid SizING AND DisPatch}

This section summarizes the results of the initial optimal sizing and dispatch study. The worst-case scenario obtained from these results defines the operating conditions for the regulation study. The sizing study optimized the sizes of the solar panel, batteries and generators, as well as the power dispatch over a period of one year using a forward-looking strategy to minimize annualized capital and fuel costs. The mathematical formulation of this nested component sizing and dispatch optimization is adopted from [23] and only the results are summarized here for brevity.

The FOB was sized for 50 soldiers, using $120 \mathrm{~kW}$ peak power and $67 \mathrm{~kW}$ average power. Solar irradiance data from Afghanistan was used. The power output from the solar panel was calculated as the product of rated power, a derating factor, and the ratio of incident solar irradiance to the peak solar irradiance of $1 \mathrm{~kW} / \mathrm{m}^{2}$. It was assumed that the derating factor of the PV array was maintained at $95 \%$ with a good maximum power point tracking control algorithm. The optimization resulted in a solar panel of $89 \mathrm{~kW}$, a total of $8.4 \mathrm{kWh}$ battery capacity (4 vehicles with $2.1 \mathrm{kWh}$ battery capacity each), and a total of $120 \mathrm{~kW}$ plug-in vehicle generator power $(30 \mathrm{~kW}$ per vehicle). It also resulted in an optimal power dispatch strategy for an entire year.

Worst case scenarios, from a regulation perspective, occur when generation or load undergoes a step (or rapid) change, introducing a sizeable mismatch between generation and consumption. Fig. 4 illustrates the hourly load and generation trajectories, for the example microgrid, for one of the days on which a worst-case transient occurred. The shaded region shows an abrupt drop in solar power. The generators were initially idling as there was sufficient solar power to supply the loads and charge the batteries. A $50 \mathrm{~kW}$ drop then occurred in the solar power (e.g., due to cloud cover), requiring the batteries and generators to support the loads and stabilize the microgrid. As can be seen in Fig. 4, the resulting optimal hourly sequence of dispatch commands involved the simultaneous increase of battery and generator power.

For the purposes of this paper, it is assumed that a drop of this magnitude could happen instantaneously and unexpectedly, for example due to a sand storm or destruction of part of the solar panel array, and hence it is referred to as the worst-case scenario. 


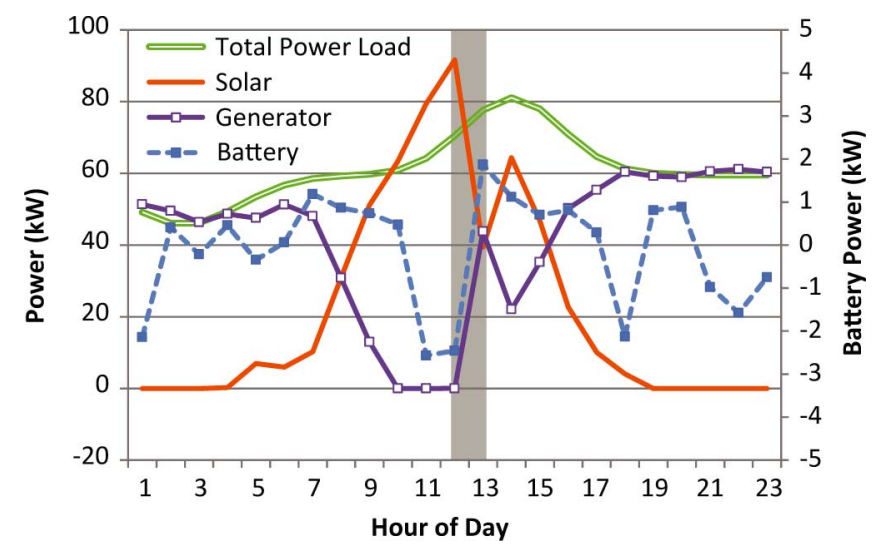

Fig. 4. The power profiles for the day that contains the worst-case scenario considered in this paper (the shaded region).

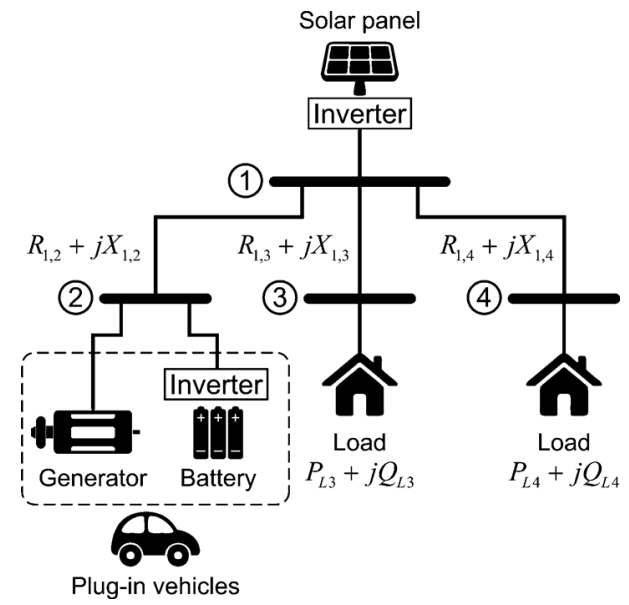

Fig. 5. Electricity grid model of the example microgrid shown in Fig. 1.

\section{MicROGRID REGULATION}

This section describes the regulation aspect of the integrated framework of Fig. 3 in detail. The worst-case scenario identified in the previous section defines the microgrid operating conditions and disturbances that is considered.

\section{A. Modeling the Microgrid}

The microgrid in Fig. 1 consists of four buses: Two of them are connected to loads; one is connected to an intermittent power source, namely, the solar panel; and one is connected to a reconfigurable energy storage and power source, namely, a group of vehicles. The grid is modeled as shown in Fig. 5. For the purposes of this paper, the vehicles are aggregated into a single generator and battery that are controlled independently. The vehicles are assumed to be always available for supporting the microgrid. Furthermore, within the short time scale of interest (i.e., seconds), the number of vehicles connected to the microgrid is assumed to remain constant, leading to a constant generator and battery size. The loads are assumed critical and hence no load-side power management (e.g., load shedding) is considered in this paper.

The solar panel and battery are interfaced with the AC microgrid through inverters. A model for the inverter-grid interface is shown in Fig. 2. The inverter is controlled to regulate the voltage $V_{t}$ at the terminal bus and active power output $P_{\text {out }}$ to the grid. This is achieved by controlling the modulation index $m$ of the inverter, which effectively controls the inverter voltage magnitude $V_{i}$ through the relationship

$$
V_{i}=m \frac{V_{\mathrm{dc}}}{V_{\mathrm{base}}},
$$

and the inverter firing angle, which effectively determines the phase angle $\delta_{i}$. In (1), $V_{\text {base }}$ is the base unit voltage that is used for normalization to allow working with the per-unit system [28], and $V_{\mathrm{dc}}$ is the DC voltage on the DC terminals of the inverter, which is established by the DC power source. The modulation index $m$ is constrained by a saturation limit $m \leq 1$, so it cannot compensate for arbitrarily low DC voltages.

This paper considers a phase-locked loop (PLL) based inverter control strategy as proposed in [24]. The PLL tracks the $\mathrm{AC}$ voltage waveform at the inverter terminals to establish an angle reference signal for the inverter firing circuitry [29]. As a by-product, it also provides an estimate of the local frequency. The power delivered to the microgrid is regulated by controlling the phase $\delta_{i}$ of the inverter-synthesized voltage waveforms relative to the PLL reference. The dynamics of the inverter controller are given by the following set of differential-algebraic equations:

$$
\begin{aligned}
\dot{m} & =K_{1}\left(V_{\text {set }}-V_{t}\right), \\
\dot{\theta} & =K_{2}\left(P_{\text {set }}-P_{\text {out }}\right), \\
\dot{a} & =K_{3}\left(\delta_{t}-\delta_{p}\right), \\
\dot{\delta}_{p} & =\omega_{p}, \\
0 & =V_{i}-\frac{m V_{\mathrm{dc}}}{V_{\text {base }}}, \\
0 & =\theta-\left(\delta_{i}-\delta_{p}\right), \\
0 & =a-\left(\omega_{p}-K_{4} \theta\right), \\
0 & =P_{\text {out }}-\frac{V_{\mathrm{dc}} I_{\text {inv }}}{P_{\text {base }}}, \\
0 & =P_{\text {out }}-\frac{V_{i} V_{t}}{X} \sin \left(\delta_{i}-\delta_{t}\right) .
\end{aligned}
$$

Equations (2a) and (2b) correspond to integral control of $V_{t}$ and $P_{\text {out }}$, where $V_{\text {set }}$ and $P_{\text {set }}$ are the desired values for $V_{t}$ and $P_{\text {out }}$, respectively.Equations (2c) and (2d) together describe the PLL dynamics, which, in addition to integral control, also involves damping due to the term $K_{4} \theta$ in the definition of the auxiliary variable $a(2 \mathrm{~g})$. The variable $\delta_{p}$ gives the PLL phase angle, and its time derivative $\omega_{p}$ provides an estimate of the deviation of system frequency from nominal. The integral action in (2c) aims to drive the difference between the PLL phase angle $\delta_{p}$ and the terminal phase angle $\delta_{t}$ to zero. Further details of this PLL model can be found in [24], [29]. Equations (2f) and (2g) define the variables $\theta$ and $a$, respectively. Equation ( $2 \mathrm{~h}$ ) describes the active power balance between the AC and DC side of the inverter, with $I_{\mathrm{inv}}$ representing the inverter DC current. Finally, (2i) gives the active power delivered to the grid. The block diagram shown in Fig. 6 illustrates the interaction between the PLL and the power control scheme.

In this study, (2) is used to model the inverters for both the solar panel and the battery. In addition to (2), the equations for 


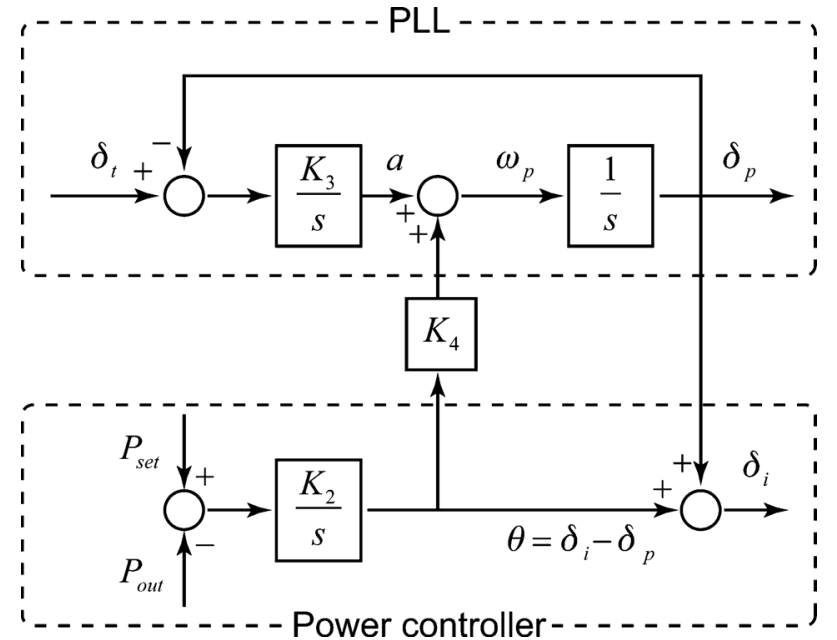

Fig. 6. The power control scheme used in this paper.

the solar panel inverter are augmented with the following exogenous input for the power setpoint

$$
P_{\text {set }}=P_{\text {solar }}(t),
$$

where $P_{\text {solar }}(t)$ is the available solar power at time $t$.

Without any control over the loads and solar panel setpoint, the only components to be considered in the power setpoint control problem are the vehicle battery and generator. This is addressed in Section III-C.

Finally, the power balance at the buses is modeled as follows. Using phasor notation, the $\mathrm{AC}$ terminal voltages are given by

$$
\mathbf{V}_{t n}=V_{t n} e^{j \delta_{t n}}, \quad n=1,2,3,4,
$$

where $n$ is an index for the terminals. The inverter internal AC bus voltages $V_{i n}$ are similarly defined. The line impedances between the terminals are expressed as

$$
\mathbf{Z}_{1, n}=R_{1, n}+j X_{1, n}, \quad n=2,3,4 .
$$

The line and inverter currents are thus obtained as

$$
\begin{aligned}
\mathbf{I}_{1, n}=\frac{\mathbf{V}_{t 1}-\mathbf{V}_{t n}}{\mathbf{Z}_{1, n}}, \quad n=2,3,4 \\
\mathbf{I}_{\text {inv } 1}=\frac{\mathbf{V}_{i 1}-\mathbf{V}_{t 1}}{j X}, \quad \mathbf{I}_{\text {inv } 2}=\frac{\mathbf{V}_{i 2}-\mathbf{V}_{t 2}}{j X} .
\end{aligned}
$$

The power balance equations are then

$$
\begin{aligned}
\mathbf{I}_{\text {inv } 1}-\mathbf{I}_{1,2}-\mathbf{I}_{1,3}-\mathbf{I}_{1,4} & =0 \\
\mathbf{V}_{t 2}\left(\mathbf{I}_{1,2}^{*}+\mathbf{I}_{\text {inv } 2}^{*}\right)+\left(P_{\text {gen }}+j Q_{\text {gen }}\right) & =0 \\
\mathbf{V}_{t 3} \mathbf{I}_{1,3}^{*}-\left(P_{L 3}+j Q_{L 3}\right) & =0 \\
\mathbf{V}_{t 4} \mathbf{I}_{1,4}^{*}-\left(P_{L 4}+j Q_{L 4}\right) & =0
\end{aligned}
$$

where asterisk denotes complex conjugate.

Based on the time-scale separation principle [30], the dynamics associated with the power electronics, which are on the order of milliseconds, are neglected when compared to the time scales of interest in this study, i.e., seconds.

\section{B. Modeling the Power Sources and Loads}

The drop in solar power (due to weather, malfunction, attack, etc.) is assumed to happen instantaneously and is thus modeled as a "worst case" step change as described by

$$
P_{\text {solar }}(t)=\left\{\begin{array}{ll}
P_{\text {solar }}^{1} & t \leq t_{1} \\
P_{\text {solar }}^{2} & t>t_{1}
\end{array} .\right.
$$

Based on the time constants reported in the literature for small diesel generators [31], the generator is assumed to have first order dynamics as given by

$$
\dot{P}_{\text {gen }}=\frac{1}{\tau_{\text {gen }}}\left(P_{\text {set }}^{\text {gen }}-P_{\text {gen }}\right)
$$

with a discussion of $P_{\text {set }}^{\text {gen }}$ provided in Section III-C.

The battery is modeled as a voltage source with an internal resistance. Within the time scale of interest (i.e., seconds), the change in the state-of-charge ( $\mathrm{SoC})$ is assumed to be negligible, and thus the $\mathrm{SoC}$ dependence of the open-circuit voltage (OCV) of the battery is neglected along with any other battery dynamics. We assume a string of batteries in series so that the nominal $V_{\mathrm{OCV}}$ at $50 \%$ charge is equal to the nominal DC bus voltage $V_{\text {base }}$. The battery is represented using the following relationship:

$$
V_{\mathrm{dc}}^{\mathrm{batt}}=V_{\mathrm{OCV}}-R_{\mathrm{batt}} I_{\mathrm{inv}},
$$

where $I_{\mathrm{inv}}$ is given by (2h), and is considered positive when the battery is discharging.

Finally, the loads are modeled as constant because they are assumed to be critical loads that need to be supplied continuously.

\section{Power Control Structure}

Within the considered framework, the only components whose power setpoints need to be managed are the vehicle battery and generator. The following feedback forms are proposed:

$$
\begin{aligned}
P_{\mathrm{set}}^{\mathrm{gen}}= & -k_{1 P} \omega_{p}-k_{1 a}\left(P_{\mathrm{gen}}-P_{\mathrm{op}}^{\mathrm{gen}}\right) \\
& -k_{1 I} \int \omega_{p} d t+P_{\mathrm{op}}^{\mathrm{gen}}, \\
P_{\mathrm{set}}^{\mathrm{batt}}= & -k_{2 P} \omega_{p}+P_{\mathrm{op}}^{\mathrm{batt}}
\end{aligned}
$$

where $P_{\mathrm{op}}$ represents the pre-disturbance operating point.

Note that the first terms on the right hand side of the equations correspond to the traditional droop control scheme [1], [6], [32]-[35]. The second and third terms in the generator controller provide secondary frequency control with a PI control strategy. However, there is a minor difference from the typical PI control approach [36] in the sense that the P and I control actions act on two separate variables. Specifically, the second term in the generator controller adds a proportional feedback from the actual power output. This helps delay the response of the generator. A disturbance will then initially be compensated for mainly by the battery, helping save fuel. However, the battery cannot compensate for a large disturbance indefinitely due to its limited energy capacity. Hence, the generator controller also includes an integral action on the frequency deviation, so that any disturbance is 
ultimately compensated for entirely by the generator. This control scheme ensures that in the post-disturbance steady state the frequency deviation is zero, thereby enabling the battery to return to its original operating condition, whereas the generator power output settles to

$$
P_{\mathrm{gen}}=P_{\mathrm{op}}^{\mathrm{gen}}-\frac{k_{1 I}}{1+k_{1 a}} \int \omega_{p} d t .
$$

It is assumed that a higher-level dispatch controller exists that will eventually be informed about the disturbance and will determine the new (optimal) operating conditions for the generator and battery.

Also note the decentralized character of the controllers in (11). The only common signal to the controllers is the frequency deviation $\omega_{p}$; the controllers are otherwise independent, use only the locally available information (i.e., frequency and local power), and do not require any communication with each other or any other component in the microgrid.

\section{Model Parameterization and Control Design}

The microgrid system described above was simulated and optimized for the parameter values given in Table I. The parameters (and later results) that are given in the per-unit system can be converted to their absolute values using the base values reported in Table I. The parameter values for the inverter grid interface and inverter control gains are taken from [24]. The loads, step change in solar power, and the operating points for the battery and generator are obtained from a worst-case scenario of the optimal sizing and dispatch study as described in Section II. The maximum allowed frequency and voltage deviations were set to $0.5 \mathrm{~Hz}$ and $5 \%$, respectively. The power regulation gains were tuned by linearizing the model around the given operating point and numerically solving an LQR problem with a constraint on frequency deviation and limits on the extent to which the power setpoints of the generator and battery could be moved. A further constraint ensures that the battery returns to its original state within $170 \mathrm{~s}$ to avoid draining the battery with subsequent energy extractions. The constraints were included with a large penalty in the optimization problem. Specifically, the objective function was formulated as

$$
J=\int_{0}^{T}\left(x^{T} Q x+u^{T} R u+C_{\lim }(x, t)\right) d t
$$

with

$$
\begin{aligned}
C_{\lim }(x, t)= & w_{1}\left(\max \left(\left|x_{1}\right|-x_{1, \mathrm{lim}}, 0\right)\right)^{2} \\
& +w_{2}\left(\max \left(u_{1}-u_{1, \mathrm{lim}}, 0\right)\right)^{2} \\
& +w_{3}\left(\max \left(\left|u_{2}\right|-u_{2, \mathrm{lim}}, 0\right)\right)^{2} \\
& +w_{4} u_{2}^{2} \mathbf{1}(t-170), \\
\mathbf{1}(t)= & \begin{cases}0 & \text { if } t<0 \\
1 & \text { else }\end{cases} \\
x_{1}= & \omega_{p}, \quad u_{1}=P_{\text {set }}^{\mathrm{gen}}-P_{\mathrm{op}}^{\mathrm{gen}}, \\
u_{2}= & P_{\text {set }}^{\text {batt }}-P_{\mathrm{op}}^{\text {batt }},
\end{aligned}
$$

where $w_{i}$ are the weights, and $\mathrm{x}_{1, \text { lim }}, u_{1, \text { lim }}$, and $u_{2, \text { lim }}$ are the limits on frequency deviation, generator power, and battery power respectively. The resulting optimization problem was solved with an unconstrained nonlinear programming solver. The weights $w_{i}$ were set equal, and progressively increased to
TABLE I

MODEL PARAMETERS

\begin{tabular}{cc||cc}
\hline \hline Parameter & Value & Parameter & Value \\
\hline$K_{1}$ & 10 & $R_{1,2}, R_{1,3}, R_{1,4}$ & $0.008 \mathrm{pu}$ \\
$K_{2}$ & 20 & $X_{1,2}, X_{1,3}, X_{1,4}$ & $0.004 \mathrm{pu}$ \\
$K_{3}$ & 20 & $P_{\text {solar }}^{1}$ & $7.949 \mathrm{pu}$ \\
$K_{4}$ & $101 / \mathrm{s}$ & $P_{\text {solar }}^{2}$ & $2.97 \mathrm{pu}$ \\
$X$ & $0.05 \mathrm{pu}$ & $P_{\text {base }}$ & $10 \mathrm{kVA}$ \\
$V_{\text {set }}$ & $1 \mathrm{pu}$ & $V_{\text {base }}$ & $110 \mathrm{~V}$ \\
$P_{L 3}$ & $3 \mathrm{pu}$ & $V_{\mathrm{dc}}^{\text {solar }}, V_{\mathrm{OCV}}$ & $480 \mathrm{~V}$ \\
$P_{L 4}$ & $3.42 \mathrm{pu}$ & $k_{1 P}$ & 0.086 \\
$Q_{L 3}, Q_{L 4}$ & 0 & $k_{1 a}$ & 1.6016 \\
$P_{\text {max }}^{\text {gen }}$ & $12 \mathrm{pu}$ & $k_{1 I}$ & 0.143 \\
$P_{\mathrm{op}}^{\mathrm{gen}}, Q_{\mathrm{gen}}$ & 0 & $k_{2 P}$ & 1.8 \\
$\tau_{\text {gen }}^{\text {ben }}$ & $1 \mathrm{~s}$ & $t_{1}$ & $1 \mathrm{~s}$ \\
$P_{\mathrm{op}}^{\text {batt }}$ & $-1.285 \mathrm{pu}$ & & \\
\hline \hline
\end{tabular}

a value of $10^{8}$ in accordance with standard penalty function methods [37].

During the control design, the battery internal resistance was neglected and the battery was assumed to be a constant voltage source. Hence, voltage constraints were also neglected in the constrained LQR formulation.

\section{REGUlATION RESUltS AND DiscuSSION}

The dynamic performance of the microgrid was explored by introducing a step change in the solar power production, as modeled by (8) with pre- and post-disturbance levels given in Table I. Figs. 7 and 8 show the frequency and voltage regulation performance of the controller. In these figures, the nonlinear microgrid model was used; however, the battery voltage was assumed to be constant to show the nominal performance of the controller. As the figures illustrate, both the frequency and voltage can be regulated successfully within the desired limits for this idealized battery voltage behavior.

Fig. 9 shows the battery and generator power trajectories during the disturbance. Initially the microgrid is stabilized using mainly the battery. Gradually the generator power is increased and, due to the integral action in the generator control scheme (11), the generator completely compensates for the solar power loss in the post-disturbance steady state, allowing the battery to return to its original charging state.

To check the impact of the constant battery voltage assumption on performance, the simulation was repeated with the battery internal resistance modeled with values varying from 0.1 to $1 \Omega$, in increments of $0.1 \Omega$. Fig. 10 shows the voltage regulation performance of the controller for select values of $R_{\text {batt }}$. Voltage constraint violations occur at terminal 4 for $R_{\text {batt }} \geq 0.3 \Omega$. Violations at other terminals are also observed for higher values of $R_{\text {batt }}$. The corresponding battery voltages are shown in Fig. 11 . The frequency regulation performance is not affected significantly by the range of $R_{\text {batt }}$ values considered and thus the corresponding plot is not shown.

The voltage sensitivity displayed in Fig. 10 can be explained by considering the power balance across the microgrid. Because 


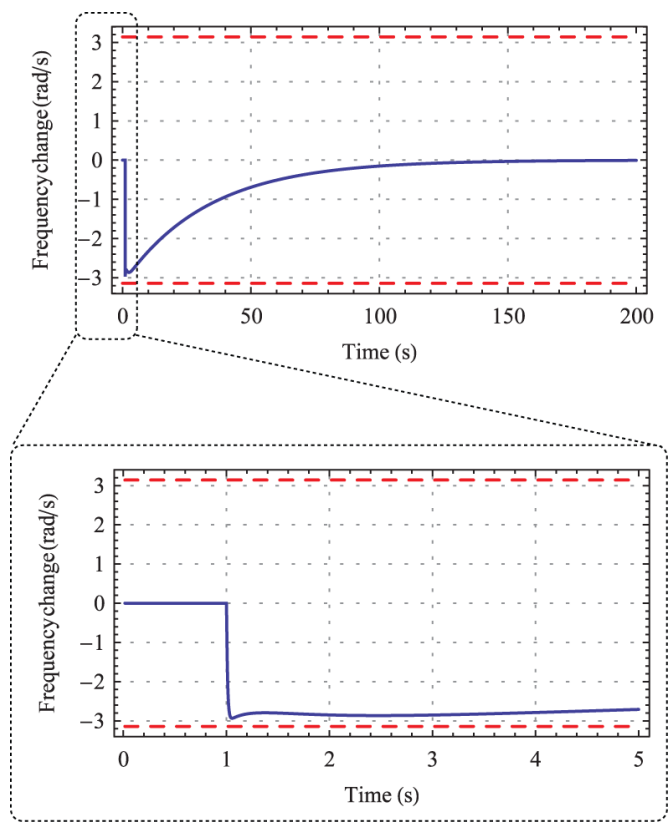

Fig. 7. Frequency regulation performance of the design assuming battery is a constant voltage source. Dashed lines represent the maximum allowed deviation limits.
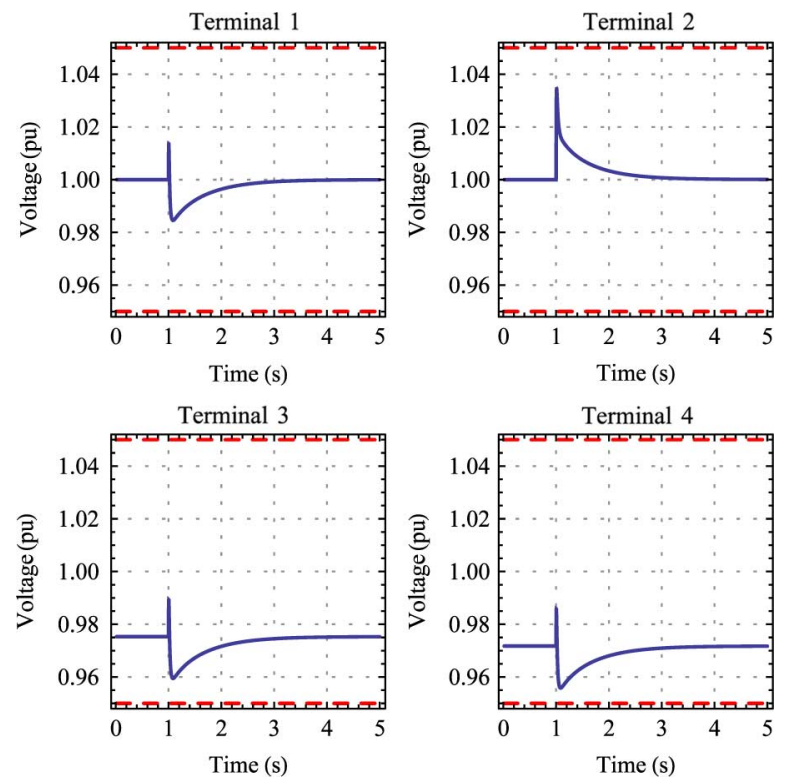

Fig. 8. Voltage regulation performance of the design for all the terminals assuming battery is a constant voltage source. Dashed lines represent the maximum allowed deviation limits.

the loads draw constant power, the total power supplied by the various sources must also remain effectively constant, though with some adjustment to account for changes in network losses. Therefore, when the solar power output reduces, the battery must take up the difference. This is not a controlled response, but rather a consequence of the power balance inherent in satisfying Kirchhoff's laws. With battery internal resistance modeled, this sudden increase in the power delivered from the battery will cause a sharp decrease in the battery terminal voltage. This is apparent in Fig. 11. Controls quickly respond to restore the microgrid AC voltages though, as shown in Fig. 10.

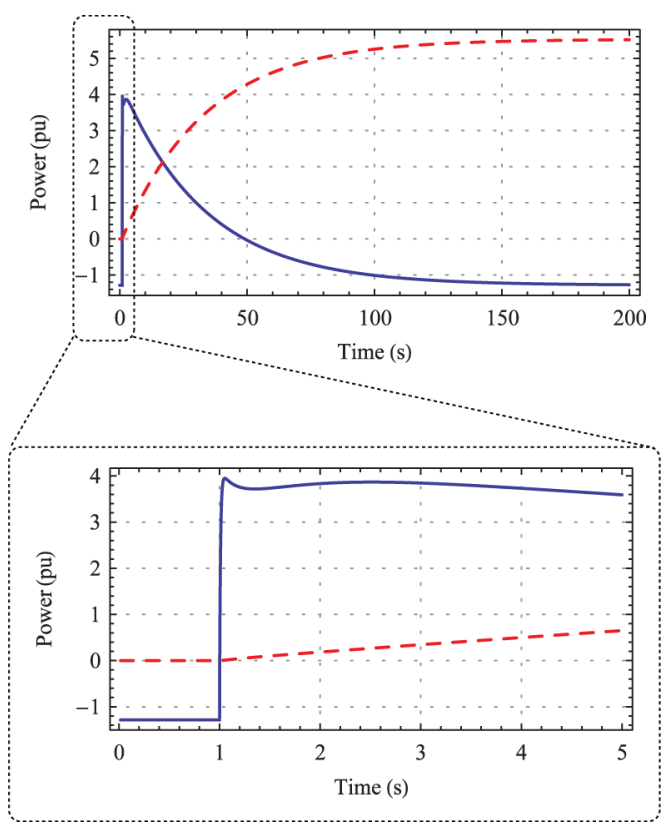

Fig. 9. Battery (solid) and generator (dashed) power.

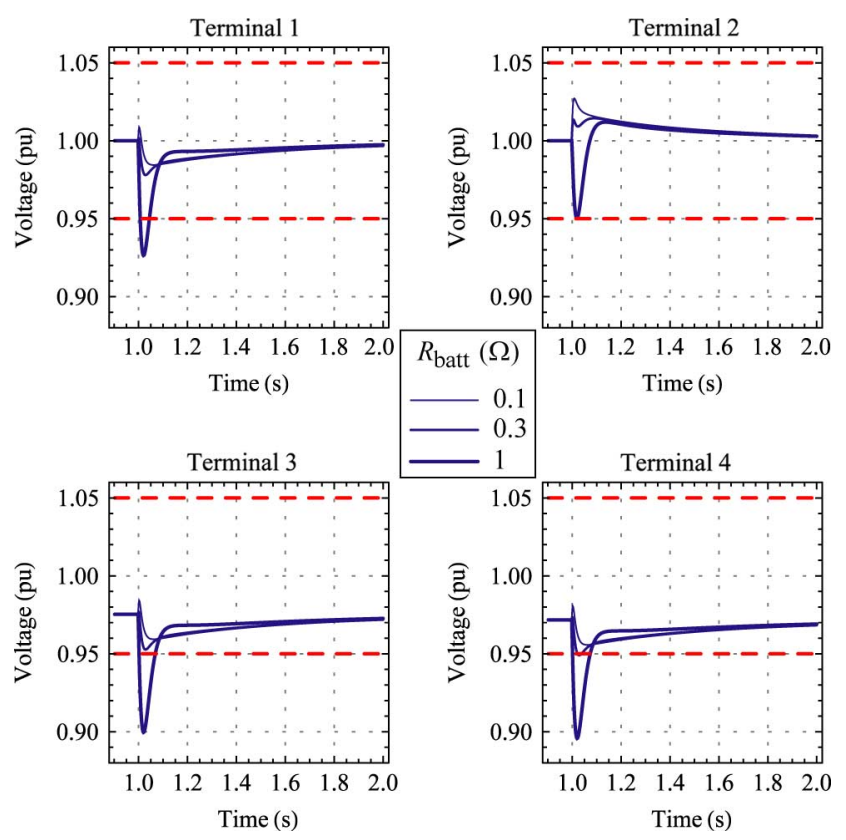

Fig. 10. Terminal voltage regulation performance of the controller for various battery internal resistance values. Dashed lines represent the maximum allowed deviation limits.

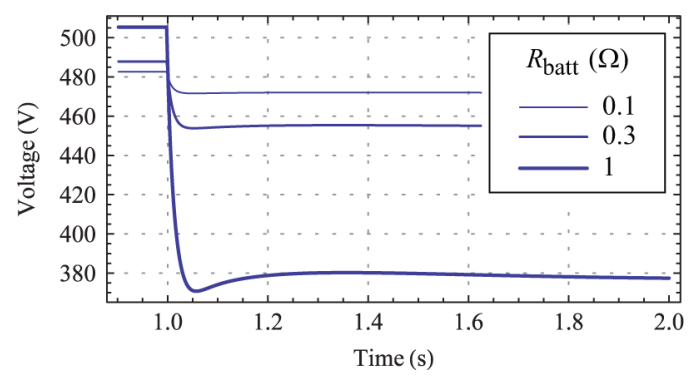

Fig. 11. Battery terminal voltage $V_{\mathrm{dc}}^{\text {batt }}$ trajectories for various battery internal resistance values. 


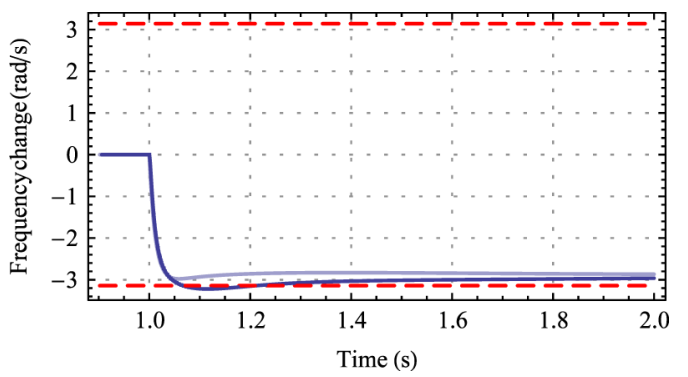

Fig. 12. Frequency regulation performance for $R_{\text {batt }}=1 \Omega$ and $K_{1}=10$ (light) versus $K_{1}=200$ (dark). Dashed lines represent the maximum allowed deviation limits.
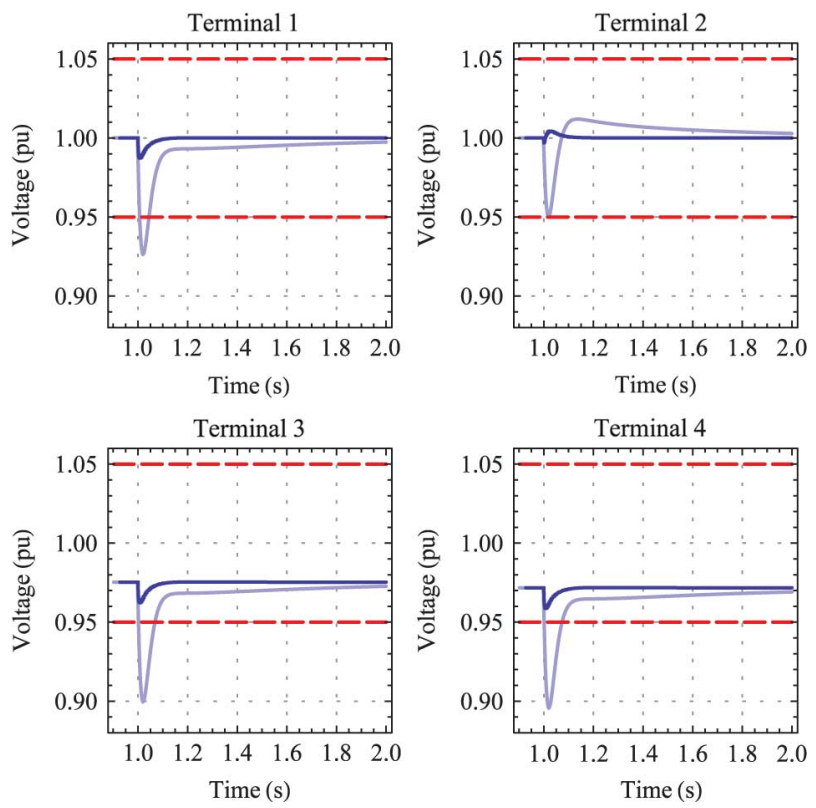

Fig. 13. Voltage regulation performance for $R_{\text {batt }}=1 \Omega$ and $K_{1}=10$ (light) versus $K_{1}=200$ (dark). Dashed lines represent the maximum allowed deviation limits.

The battery power needed to ensure satisfactory recovery from the loss of solar power is around $40 \mathrm{~kW}$ (Fig. 9). The maximum theoretical battery power available is

$$
P_{\max }^{\text {batt }}=\frac{V_{\mathrm{OCV}}^{2}}{4 R_{\text {batt }}}
$$

which gives $P_{\max }^{\text {batt }}=57.6 \mathrm{KW}$ for $R_{\mathrm{batt}}=1 \Omega$. Hence, the battery is capable of providing the power needed, but cannot maintain the terminal voltages within desired limits.

The high sensitivity of voltage and low sensitivity of frequency to battery voltage fluctuations may seem to indicate that the voltage and frequency regulation problems are decoupled, implying the voltage control gain $K_{1}$ of the inverters may be increased to reduce the sensitivity of the voltages without affecting frequency regulation performance. However, Figs. 12 and 13 illustrate that this is not necessarily the case. These figures compare the regulation performance for the original value of $K_{1}=10$ and for a high gain of $K_{1}=200$. In both cases $R_{\text {batt }}$ was set to $1 \Omega$. The higher controller gain greatly improves the voltage regulation performance and all terminal voltages are well within the desired limits. However, the frequency constraint is violated. To understand this frequency excursion, recall that the inverter control design, given by (2) and (11), introduces a droop characteristic that inversely couples power output and frequency ${ }^{1}$. The higher gain $K_{1}=200$ causes a higher overshoot in the power output than was the case with $K_{1}=10$. This larger power overshoot causes frequency to transiently undershoot its allowable limit, as shown in Fig. 12.

Therefore, it is critical to consider the voltage-current characteristics of the battery carefully and compensate for undesirable voltage drops either in the control design (e.g., by taking the battery characteristics into account and considering both frequency and voltage regulation together), or in the battery design (e.g., by changing battery chemistry, increasing battery size, and/or adding capacitors in parallel). The next section further considers the latter option, and in doing so closes the loop between the regulation and component sizing problems.

\section{IMPLICATIONS FOR COMPONENT SIZING}

The simulations presented in Section IV have been performed without considering any particular battery chemistry. However, in practice, the battery chemistry and configuration, i.e., number of battery cells in series and parallel, will dictate the internal resistance of the battery pack. The chemistry and configuration will also determine the energy capacity of the battery. Hence, it is of interest to study the extent to which regulation considerations can impact the energy capacity, i.e., the sizing, of the battery. This section considers the original inverter and setpoint control designs of Section III-D. Under that condition, Fig. 11 showed that a battery with $R_{\text {batt }}=0.1 \Omega$ yields satisfactory regulation performance.

As a representative battery for hybrid electric vehicle applications, an $11.1 \mathrm{~V}$ (3-cell) $5 \mathrm{Ah} \mathrm{Li}$-polymer battery is considered. The internal resistance of such a battery has been identified as $48.3 \mathrm{~m} \Omega$ [38]. To achieve a $480 \mathrm{~V}$ DC voltage supply for the inverter, 44 of these batteries should be connected in series. Furthermore, to achieve the $8.4 \mathrm{kWh}$ battery capacity required by the optimal sizing solution mentioned in Section II, 4 parallel connections are required. This brings the capacity of the battery pack to $9.8 \mathrm{kWh}$.

On the other hand, with 44 series and 4 parallel connections, the total equivalent internal resistance of the battery pack is $0.53 \Omega$. This is above the desired value of $0.1 \Omega$ and in the range that leads to undesired voltage fluctuations as shown in Fig. 11. To bring the battery internal resistance down to $0.1 \Omega$, the number of parallel connections needs to be increased to 22 , which in turn implies that the battery size increases from $9.8 \mathrm{kWh}$ to $53.7 \mathrm{kWh}$.

This example highlights an important coupling between the component sizing and regulation problems. It shows that an optimal sizing study that considers only the energy capacity of the battery, e.g., [17]-[19], [22], [23], can potentially undersize the battery.

This undersizing issue is not only important for the battery, but also for the sizing of other components in the microgrid. As an example, if the $53.7 \mathrm{kWh}$ minimum battery size is introduced into the optimization framework of Section II as an additional constraint, the solar panel size reduces from $89 \mathrm{~kW}$ to $87 \mathrm{~kW}$ to partially offset the increased capital cost of the batteries. Moreover, the annualized capital cost increases from

\footnotetext{
${ }^{1}$ Because inverter-based microgrids do not have synchronous generation, there is no natural relationship between frequency and power balance. This familiar behavior must be established by control action.
} 
TABLE II

COMParison OF Optimal SizING With AND Without Regulation CONSTRAINT

\begin{tabular}{ccc}
\hline \hline & $\begin{array}{c}\text { Optimal sizing } \\
\text { without regulation } \\
\text { constraint }\end{array}$ & $\begin{array}{c}\text { Optimal sizing } \\
\text { with regulation } \\
\text { constraint }\end{array}$ \\
\hline Solar panel size & $89 \mathrm{~kW}$ & $87 \mathrm{~kW}$ \\
Battery capacity & $8.4 \mathrm{kWh}$ & $57.2 \mathrm{kWh}$ \\
$\begin{array}{c}\text { Capital cost } \\
\text { (annualized) }\end{array}$ & $\$ 91,500$ & $\$ 94,100$ \\
Fuel use (annual) & $143.8 \mathrm{~kL}$ & $146.1 \mathrm{~kL}$ \\
\hline \hline
\end{tabular}

$\$ 91500$ to $\$ 94100$, and the annual fuel use is estimated to increase from $143.8 \mathrm{~kL}$ to $146.1 \mathrm{~kL}$. These results are summarized in Table II. The increased fuel use is due to the reduced solar panel power, showing a tradeoff of meeting the regulation requirements.

It is important to re-emphasize that these results are presented as an illustration rather than an ultimate solution. For example, the dynamics of the battery have been neglected for simplicity, including the dependence of OCV on SoC. While this may be an appropriate assumption over the short time scale of interest in this paper, the dependence of OCV on SoC could be a significant factor in other circumstances. If so, it would be important to consider the battery chemistry, as different types of batteries have different OCV-SoC characteristics. For chemistries such as Li-ion, the OCV-SoC curve is quite flat in the normal operating region, i.e., the $\mathrm{OCV}$ is rather insensitive to changes in SoC. In such cases, it would be reasonable to expect that regulation capability is similarly insensitive to SoC. Other chemistries may display a more pronounced dependence of OCV on SoC that could affect the regulation capability.

Other types of storage could also be considered, for example using capacitors to augment or replace the batteries. However, such analysis is beyond the scope of this paper and is left as future work. To reiterate, the main goal of this paper has been to identify the regulation-sizing coupling, emphasize its importance, and highlight the need for an integrated design approach of the form proposed in the paper. Hence, the particular solution considered in this section, that of increasing the battery size, is not to be interpreted as the only or optimal solution.

\section{SUMMARY AND CONCLUSION}

The paper considered a conceptual military microgrid that operates autonomously, i.e., without connection to a larger power grid. A solar panel, along with vehicle batteries and generators provide the power, while loads are assumed to be constant and uncontrollable. Leveraging a decentralized, phase-lockedloop based inverter control strategy, this paper has considered a power setpoint control algorithm for the batteries and generators. The controller is tuned assuming the battery voltage is constant. The impact of this assumption is tested by considering a range of values for the battery internal resistance.

The results show that the battery internal resistance can affect the performance of both frequency and voltage regulation. Thus, an effective inverter-based control design framework should consider both regulation problems together, as well as the voltage-current characteristics of the DC sources.
The results further illustrate the impact of regulation considerations on the sizing of the components in the microgrid. This is illustrated by considering an increase in the battery size as one potential solution. Hence, an important coupling between the component sizing and regulation problems is shown. This coupling implies that the typical approach, where the two problems are considered separately due to their disparate time scales, may lead to unsatisfactory designs. It is concluded that microgrid sizing, dispatch, and regulation problems should be considered concurrently using an integrated design framework.

\section{REFERENCES}

[1] R. Lasseter, A. Akhil, C. Marnay, J. Stephens, J. Dagle, R. Guttromson, A. S. Meliopoulous, R. Yinger, and J. Eto, Integration of distributed energy resources: The CERTS microgrid concept U.S. Department of Energy and California Energy Commission, LBNL-50829, 2002.

[2] H.-W. Zhao and T.-t. Wu, "Review of distributed generation based microgrid technology," Proc. CSU-EPSA, vol. 20, no. 1, pp. 121-128, 2008.

[3] M. A. Sofla and G. B. Gharehpetian, "Dynamic performance enhancement of microgrids by advanced sliding mode controller," Int. J. Electr. Power Energy Syst., vol. 33, no. 1, pp. 1-7, 2011.

[4] D. Pudjianto, C. Ramsay, and G. Strbac, "Microgrids and virtual power plants: Concepts to support the integration of distributed energy resources," Proc. Inst. Mech. Eng. Part A, J. Power Energy, vol. 222, no. 7, pp. 731-741, 2008.

[5] J. A. P. Lopes, S. A. Polenz, C. L. Moreira, and R. Cherkaoui, "Identification of control and management strategies for LV unbalanced microgrids with plugged-in electric vehicles," Elect. Power Syst. Res., vol. 80, no. 8, pp. 898-906, 2010.

[6] J. A. P. Lopes, C. L. Moreira, and A. G. Madureira, "Defining control strategies for microgrids islanded operation," IEEE Trans. Power Syst., vol. 21, no. 2, pp. 916-924, 2006.

[7] T. Logenthiran, D. Srinivasan, and A. M. Khambadkone, "Multi-agent system for energy resource scheduling of integrated microgrids in a distributed system," Elect. Power Syst. Res., vol. 81, no. 1, pp. 138-148, 2011.

[8] N. W. A. Lidula and A. D. Rajapakse, "Microgrids research: A review of experimental microgrids and test systems," Renewable Sustainable Energy Rev., vol. 15, no. 1, pp. 186-202, 2011.

[9] N. Jayawarna and M. Barnes, "Study of a microgrid with vehicle-to-grid sources during network contingencies," Intell. Autom. Soft Comput., vol. 16, no. 2, pp. 289-302, 2010.

[10] J. M. Guerrero, J. Matas, L. G. De Vicuna, M. Castilla, and J. Miret, "Wireless-control strategy for parallel operation of distributed-generation inverters," IEEE Trans. Ind. Electron., vol. 53, pp. 1461-1470, 2006.

[11] T. C. Green and M. Prodanovic, "Control of inverter-based microgrids,” Elect. Power Syst. Res., vol. 77, no. 9, pp. 1204-1213, 2007.

[12] "Deployed energy storage (DES) program," DARPA-BAA-11-53, 2011.

[13] D. S. Eady, S. B. Siegel, R. S. Bell, and S. H. Dicke, "Sustain the mission project: Casualty factors for fuel and water resupply convoys," Army Environmental Policy Inst., Final Tech. Rep., NSN 7540-01280-5500, 2009.

[14] A. Gupta, R. P. Saini, and M. P. Sharma, "Steady-state modelling of hybrid energy system for off grid electrification of cluster of villages," Renewable Energy, vol. 35, no. 2, pp. 520-535, 2010.

[15] A. J. del Real, M. D. Galus, C. Bordons, and G. Andersson, "Optimal power dispatch of energy networks including external power exchange," in Proc. Eur. Control Conf., Budapest, Hungary, 2009.

[16] A. D. Hawkes and M. A. Leach, "Modelling high level system design and unit commitment for a microgrid," Appl. Energy, vol. 86, no. 7-8, pp. 1253-1265, 2009.

[17] M. Stadler, C. Marnay, A. Siddiqui, J. Lai, B. Coffey, and H. Aki, "Effect of heat and electricity storage and reliability on microgrid viability: A study of commercial buildings in California and New York states," Ernest Orlando Lawrence Berkeley Nat. Laboratory, LBNL1334E-2009, 2009.

[18] H. Asano, H. Watanabe, and S. Bando, "Methodology to design the capacity of a microgrid," in Proc. IEEE Int. Conf. Syst. Syst. Eng. (SOSE), San Antonio, TX, USA, 2007. 
[19] T. Lambert, P. Gilman, and P. Lilienthal, "Micropower system modeling with HOMER," in Integration of Alternative Sources of Energy, F. A. Farret and M. G. Simoes, Eds. New York: Wiley, 2006.

[20] S. Lu, N. B. Schroeder, H. M. Kim, and U. V. Shanbhag, "Hybrid power/energy generation through multidisciplinary and multilevel design optimization with complementarity constraints," J. Mech. Design, vol. 132, no. 10, pp. 101007-101019, 2010.

[21] M. Arnold, R. R. Negenborn, G. Andersson, and B. De Schutter, "Multi-area predictive control for combined electricity and natural gas systems," in Proc. Eur. Control Conf., Budapest, Hungary, 2009.

[22] D. L. Peters, A. R. Mechtenberg, J. W. Whitefoot, and P. Y. Papalambros, "Model predictive control of a microgrid with plug-in vehicles: Error modeling and the role of prediction horizon," in Proc. ASME Dyn. Syst. Control Conf., Arlington, VA, USA, 2011.

[23] J. W. Whitefoot, A. R. Mechtenberg, D. L. Peters, and P. Y. Papalambros, "Optimal component sizing and forward-looking dispatch of an electrical microgrid for energy storage planning," in Proc. ASME Int. Design Eng. Tech. Conf. Comput. Inf. Eng. Conf. (IDETC/CIE), Washington, DC, USA, 2011.

[24] I. A. Hiskens and E. M. Fleming, "Control of inverter-connected sources in autonomous microgrids," in Proc. Amer. Control Conf., 2008, pp. 586-590.

[25] M. Surprenant, I. Hiskens, and G. Venkataramanan, "Phase locked loop control of inverters in a microgrid," in Proc. IEEE Energy Convers. Congr. Expo., Phoenix, AZ, USA, 2011, pp. 667-672.

[26] K. De Brabandere, B. Bolsens, J. Van den Keybus, A. Woyte, J. Driesen, and R. Belmans, "A voltage and frequency droop control method for parallel inverters," IEEE Trans. Power Electron., vol. 22, no. 4, pp. 1107-1115, 2007.

[27] T. Ersal, C. Ahn, I. A. Hiskens, H. Peng, and J. L. Stein, "Impact of controlled plug-in EVs on microgrids: A military microgrid example," in Proc. IEEE Power Energy Soc. Gen. Meet., Detroit, MI, USA, 2011.

[28] J. D. Glover, M. S. Sarma, and T. J. Overbye, Power System Analysis and Design. New York: Thomson, 2008.

[29] D. Abramovitch, "Phase-locked loops: A control centric tutorial," in Proc. Amer. Control Conf., Anchorage, AK, USA, 2002, vol. 1, pp. $1-15$.

[30] T. Ersal, H. K. Fathy, L. S. Louca, D. G. Rideout, and J. L. Stein, "A review of proper modeling techniques," J. Dyn. Syst. Measure. Control, vol. 130, no. 6, p. 061008, 2008.

[31] M. Dewadasa, A. Ghosh, and G. Ledwich, "Dynamic response of distributed generators in a hybrid microgrid," in Proc. IEEE PES Gen. Meet., Detroit, MI, USA, 2011.

[32] J. A. P. Lopes, C. C. L. Moreira, and F. O. Resende, "Microgrids black start and islanded operation," in Proc. 15th Power Syst. Comput. Conf., Liege, Belgium, 2005.

[33] N. J. Gil and P. A. Lopes, "Hierarchical frequency control scheme for islanded multi-microgrids operation," in Proc. IEEE Lausanne POWERTECH, 2007, pp. 473-478.

[34] R. H. Lasseter and P. Paigi, "Microgrid: A conceptual solution," in Proc. IEEE Power Electron. Specialists Conf., 2004, vol. 6, pp. 4285-4290.

[35] R. H. Lasseter, "CERTS microgrid," in Proc. IEEE Int. Conf. Syst. Syst. Eng., 2007.

[36] Y. Xu, H. Li, and L. M. Tolbert, "Inverter-based microgrid control and stable islanding transition," in Proc. IEEE Energy Convers. Congr. Expo., Raleigh, NC, USA, 2012, pp. 2374-2380.

[37] J. Nocedal and S. J. Wright, Numerical Optimization, 2nd ed. New York: Springer, 2006.

[38] T. Hu, B. Zanchi, and J. Zhao, "Determining battery parameters by simple algebraic method," in Proc. Amer. Control Conf., San Francisco, CA, USA, 2011, pp. 3090-3095.

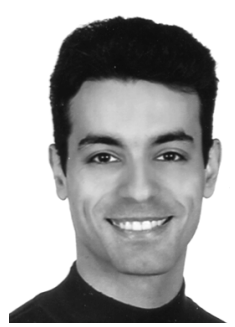

Tulga Ersal received the B.S.E. degree from the Istanbul Technical University, Istanbul, Turkey, in 2001, and the M.S. and Ph.D. degrees from the University of Michigan, Ann Arbor, MI, USA, in 2003 and 2007, respectively, all in mechanical engineering.

He is currently an Assistant Research Scientist in the Department of Mechanical Engineering, University of Michigan. His research interests include modeling, simulation, and control of dynamic systems, and model order and structure reduction, with applications to energy systems, multibody dynamics, vehicle systems, and biomechanics.
Dr. Ersal is a member of the ASME. He is the recipient of Dean's and President's Awards at the Istanbul Technical University, the Siemens Excellence Award, and three merit fellowships at the Istanbul Technical University and the University of Michigan. He also received the semi-plenary paper award at the 2012 Dynamic Systems and Control Conference.

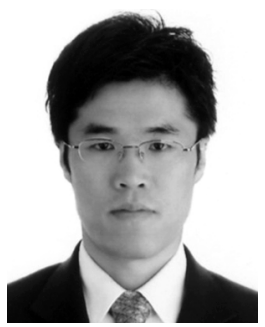

Changsun Ahn received the B.S. and M.S. degrees from Seoul National University, Korea, in 1999 and 2005, respectively, and the Ph.D. degree from the University of Michigan, Ann Arbor, MI, USA, in 2011, all in mechanical engineering.

$\mathrm{He}$ is a senior researcher in Korea Institute of Machinery and Materials, Daejon, Korea. His research interests include the fields of automotive control/estimation and energy system control. Recently, he focuses on the energy flow control of smart grids and microgrids especially having plug in electric vehi-

cles.

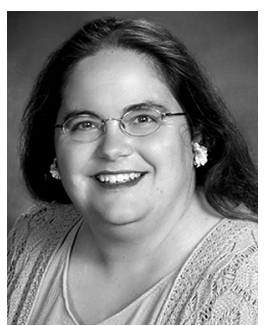

Diane L. Peters (M'11) received the B.S. degree in mechanical engineering from the University of Notre Dame, Notre Dame, IN, USA, in 1993; the M.S. degree in mechanical engineering from the University of Illinois at Chicago, Chicago, IL, USA, in 2000 and the $\mathrm{Ph} . \mathrm{D}$. degree in mechanical engineering from the University of Michigan, Ann Arbor, MI, USA, in 2010.

From 1993 to 1995 she was an Engineer at A. B. Dick Company. She was a Designer from 1995 to 1998, and a Senior Designer from 1998 to 1999 , at Mid-West Automation Systems. She was a Project Engineer at Western Printing Machinery from 1999 to 2006, a Postdoctoral Research Fellow at the University of Michigan from 2010 to 2011, and since 2011 she has been a Senior Control Systems Engineer for LMS International, a Siemens Business. Her research interest is in the coupling between design and control in mechatronic systems.

Dr. Peters is a senior life member of the Society of Women Engineers (SWE) and a member of ASME, ASEE, and ASM.

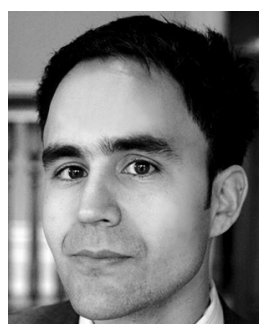

John W. Whitefoot received the B.S.E. and M.S.E. degrees in mechanical engineering from the University of Michigan, Ann Arbor, MI, USA, in 2001 and the $\mathrm{Ph} . \mathrm{D}$. degree in mechanical engineering from the University of Michigan in 2012.

From 2003 to 2008, he was a Research Manager at AGC Automotive North America R\&D. Since 2012 he has been an engineer in the Rulemaking Division of the National Highway Traffic Safety Administration in Washington, DC, USA. His research interests include technologies for improving the fuel economy of light-duty and heavy-duty vehicles, with particular emphasis on vehicle electrification.

Mr. Whitefoot's awards and honors include the Secretary's Team Award (U.S. Department of Transportation) in 2012, the ASME IDETC Best Paper Award in 2011, and the Dwight D. Eisenhower Transportation Fellowship in 2009.

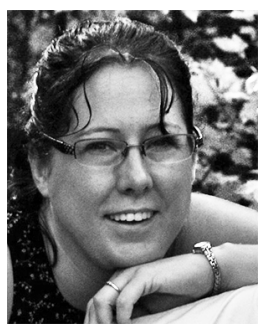

Abigali R. Mechtenberg received the B.S. and M.S. degrees in physics from Texas A\&M University, College Station, TX, USA, in 1997, the M.Ed at University of California, Santa Barbara, CA, USA, in educational psychology in 1999 and then Ph.D. degree in Applied Physics at University of Michigan, Ann Arbor, MI, USA, from 2005 to 2009 .

From 2009 to 2010, she was a Research Fellow in the Mechanical Engineering Department at the University of Michigan. Then in 2011, she was a Visiting Assistant Professor at Clark University. Since 2012, she has been a Visiting Assistant Professor at Franklin W. Olin College of Engi- 
neering in Energy Systems. Her research focuses on understanding that national security and environmental stability rely on criteria optimization of energy production, storage and consumption within a complex system of networks: social, natural and engineered.

Dr. Mechtenberg's awards and honors include the Design Automation Committee as part of ASME's IDETC Best Paper of the Year Award and SAME Sustainability Award for Army FOB Energy Quality Analysis, both in 2011.

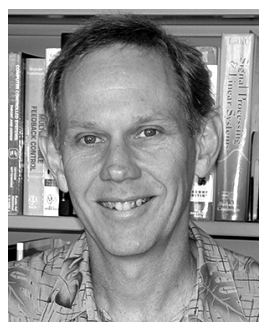

Ian A. Hiskens ( S'77-M'80-SM'96-F'06) received the B.Eng. degree in electrical engineering and the B.App.Sc. degree in mathematics from the Capricornia Institute of Advanced Education, Rockhampton, Australia, in 1980 and 1983, respectively, and the Ph.D. degree in electrical engineering from the University of Newcastle, Australia, in 1991.

$\mathrm{He}$ is the Vennema Professor of Engineering in the Department of Electrical Engineering and Computer Science, University of Michigan, Ann Arbor, MI, USA. He has held prior appointments in the Queensland electricity supply industry, and various universities in Australia and the United States. His major research interests lie in the area of power system analysis, in particular system dynamics and control, and security assessment. His recent activity has focused largely on integration of new forms of generation and load. Other research interests include nonlinear and hybrid dynamical systems.

Dr. Hiskens is actively involved in various IEEE societies, and is Treasurer of the IEEE Systems Council. He is a Fellow of Engineers Australia and a Chartered Professional Engineer in Australia.

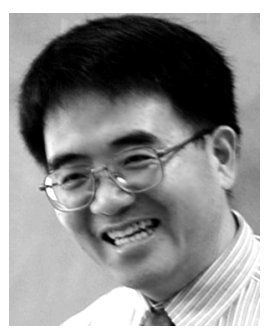

Huei Peng received his Ph.D. in mechanical engineering from the University of California, Berkeley, CA, USA, in 1992.

He is now a Professor at the Department of Mechanical Engineering at the University of Michigan, Ann Arbor, MI, USA. His research interests include adaptive control and optimal control, with emphasis on their applications to vehicular and transportation systems. His current research focuses include design and control of hybrid vehicles and vehicle active safety systems. He is currently the U.S. Director of the DOE sponsored Clean Energy Research Center-Clean Vehicle Consortium, which supports more than 30 research projects related to the development and analysis of clean vehicles in U.S. and China. He also leads an educational project funded by DOE to develop 10 undergraduate and graduate courses including three laboratories courses focusing on transportation electrification. He has more than 200 technical publications, including 90 in referred journals and transactions. His h-index is 42 according to the Google scholar analysis, the total number of citations to his work is more than 6500 .

Dr. Peng has been an active member of the Society of Automotive Engineers (SAE) and the American Society of Mechanical Engineers (ASME). He is currently the chair of the ASME Dynamic System and Control Division. He served as an Associate Editor for the IEEE/ASME Transactions on Mechatronics from 1998-2004 and for the ASME Journal of Dynamic Systems, Measurement and Control from 2004-2009. He received the National Science Foundation (NSF) Career award in 1998. He is an ASME Fellow. He is a ChangJiang Scholar at the Tsinghua University of China.

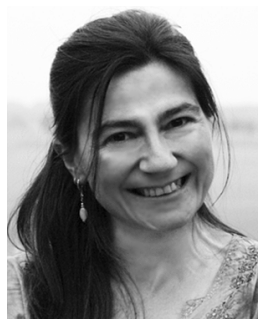

Anna G. Stefanopoulou (F'09) is a professor of Mechanical Engineering and the Director of the Automotive Research Center at the University of Michigan, Ann Arbor, MI, USA. She was an Assistant Professor (1998-2000) at the University of California, Santa Barbara, CA, USA, and a technical specialist (1996-1997) at Ford Motor Company. She is an ASME and an IEEE Fellow. She has a book, ten U.S. patents, 5 best paper awards, and more than 200 publications on estimation and control of internal combustion engines and electrochemical processes such as fuel cells and batteries.

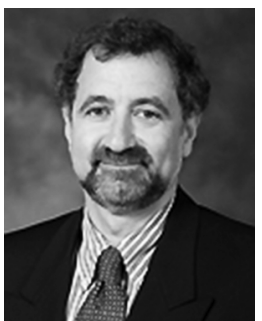

Panos Y. Papalambros is the Graham Professor of Engineering; Professor of Mechanical Engineering; Architecture; and Art and Design, at the University of Michigan, Ann Arbor, MI, USA. He holds a diploma from the National Technical University of Athens and M.S. and Ph.D. degrees from Stanford University, Stanford, CA, USA. He conducts research in design science and systems optimization, with applications to automotive systems and sustainable products. He has co-authored the textbook Principles of Optimal Design: Modeling and Computation. He is a Fellow of ASME and SAE, and recipient of the JSME Systems and Design Achievement, ASME Design Automation, ASME Machine Design, and ASME Spira Outstanding Design Educator Awards.

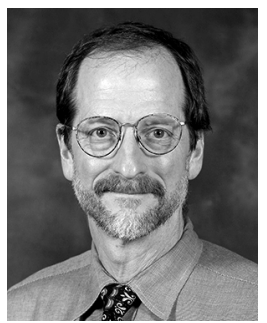

Jeffrey L. Stein received the B.S. degree in premedical studies from the University of Massachusetts, Amherst, MA, USA, in 1973, and the S.B., S.M., and $\mathrm{Ph} . \mathrm{D}$. degrees in mechanical engineering from the Massachusetts Institute of Technology, Cambridge, MA, USA, in 1976, 1976, and 1983, respectively.

Since 1983 he has been with the University of Michigan, Ann Arbor, MI, USA, where he is currently a Professor of Mechanical Engineering. He served as the Area Editor of Simulation: Transactions of The Society for Modeling and Simulation International (2007-2009), and the Associate Editor of the ASME Journal of Dynamic Systems Measurement and Control (1991-1996). He is currently an Associate Editor for Simulation Modelling Practice and Theory. His research interests include computer based modeling and simulation tools for system design and control, with applications to vehicle-to-grid integration, vehicle electrification, conventional vehicles, machine tools, and lower leg prosthetics. $\mathrm{He}$ has particular interest in algorithms for automating the development of proper dynamic mathematical models, i.e., minimum yet sufficient complexity models with physical parameters.

Dr. Stein is a fellow of the ASME, and also a member of the NSPE, SME, SAE, ASEE, SCS, and honorary societies Phi Beta Kappa, Pi Tau Sigma, Phi Kappa Phi, and Sigma Xi. He is also a Professional Engineer. 